\title{
Effect of noble metal adhesive systems on bonding between an indirect composite material and a gold alloy
}

\author{
Kiyoshi Nagano $^{\S}$, Naomi Tanoue ${ }^{\dagger}$, Mitsuru Atsuta ${ }^{\S}$, Hiroyasu Koizumi ${ }^{\ddagger}, \mathbb{I}$ \\ and Hideo Matsumura $\$$

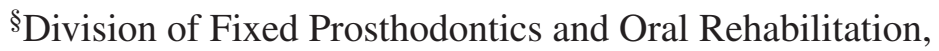 \\ Nagasaki University Graduate School of Biomedical Sciences, Nagasaki, Japan \\ ${ }^{\dagger}$ Department of Specialized Dentistry, Nagasaki University Hospital of Medicine and Dentistry, \\ Nagasaki, Japan \\ †Department of Crown and Bridge Prosthodontics, Nihon University School of Dentistry, \\ Tokyo, Japan \\ IIDivision of Advanced Dental Treatment, Dental Research Center, \\ Nihon University School of Dentistry, Tokyo, Japan
}

(Received 4 August and accepted 17 September 2004)

\begin{abstract}
In this study, the bond strength between an indirect composite and a gold alloy was determined for the purpose of evaluating noble metal bonding systems. A single liquid primer designed for conditioning noble metal alloys (Infis Opaque Primer) and tri-n-butylborane-initiated adhesive resins (SuperBond C \& B), with or without the powder component, were assessed. Cast gold alloy disks (Casting Gold type IV) were air-abraded with alumina, followed by six surface preparations, and were then bonded with a light-activated composite material (New Metacolor Infis). Shear testing was performed both before and after thermocycling for evaluation of bond durability. The results showed that three primed groups improved post-thermocycling bond strengths compared to each of the corresponding unprimed groups $(P<0.01)$. The bond strength was reduced for all six groups by the application of thermocycling $(P<0.01)$. After thermocycling, the group primed with the Infis Opaque Primer material and bonded with the Super-Bond C \& B resin exhibited the greatest bond strength (23.4 MPa). The Infis Opaque Primer and Super-Bond

Correspondence to Dr. Hideo Matsumura, Department of Crown and Bridge Prosthodontics, Nihon University School of Dentistry, 1-8-13 Kanda-Surugadai, Chiyoda-ku, Tokyo 101-8310, Japan Tel: +81-3-3219-8145

Fax: +81-3-3219-8351

E-mail: matsumura@dent.nihon-u.ac.jp
\end{abstract}

bonding system increased the post-thermocycling bond strength of the control group by a factor of approximately ten. This simple technique is applicable in the fabrication of composite veneered restorations and cone-telescope dentures. (J. Oral Sci. 46, 235-239, 2004)

Key words: alloy; bonding; noble metal; opaque resin; thiol; thione.

\section{Introduction}

The use of adhesive agents in the fabrication of composite veneered restorations has increased substantially. This trend is mainly attributed to the development of chemical bonding systems for dental casting alloys. A tri-nbutylborane-initiated (TBB)-initiated adhesive opaque resin was developed for bonding base metal alloys (1). The TBB-initiated opaque resin (Super-Bond Opaque, Sun Medical., Shiga, Japan) contains poly (methyl methacrylate) (PMMA)-coated titanium dioxide as a pigment, and 4methacryloyloxyethyl trimellitate anhydride (4-META) as a carboxylic functional monomer. A single liquid primer for conditioning noble metal alloys was thereafter developed in the 1980's. The primer, currently identified as the VPrimer or Infis Opaque Primer material (Sun Medical) contains 6-(4-vinylbenzyl- $n$-propyl)amino-1,3,5-triazine2,4-dithiol, -dithione tautomer (VTD) (2) in acetone. The 
bonding characteristics of the V-Primer material have been evaluated both in the laboratory (3-17) and clinically (18-23). In addition, the bond strengths between a lightactivated indirect composite and dental casting alloys primed with the V-Primer agent have been evaluated in combination with the Super-Bond Opaque resin (3). The results demonstrated that the V-Primer agent effectively enhanced the bond strength to noble alloys and that the bond strength of silver-palladium casting alloy was more durable than that of gold alloy. Clinical reports have demonstrated a durable bond for the V-Primer material when used together with Super-Bond resin (18,19,21-23). Although the V-Primer and Infis Opaque Primer materials considerably enhanced the bond strength to noble alloys when combined with the TBB initiator, the priming effect was not particularly good when the materials were used together with light-activated or benzoyl peroxide-amineinitiated materials $(8,13,17)$.

Application of metal priming agent together with the TBB-initiated resin at the interface between the veneering composite and the metal substructure is certainly acceptable, particularly in the case of cone-telescope dentures, in which continuous loading is applied to the external facing crowns. However, the thickness of the bonding agent and opaque resin should be controlled so that it is as thin as possible for color reproducibility of the veneering composite. Although application of the TBB opaque resin to the coloring agent has been reported $(1,3)$, the information concerning the bonding between noble metal alloys and light-activated opaque resins using the TBB resin as a bonding agent is limited. The purpose of the current study is to evaluate the effects of a metal priming agent, an unfilled TBB resin bonding agent, and a TBB-initiated opaque resin on the durability of the bond between a lightactivated indirect composite material and a gold casting alloy.

\section{Materials and Methods}

A single liquid metal conditioner designed for priming noble metal alloys (Infis Opaque Primer, Sun Medical) was assessed. The primer contains VTD thione monomer in acetone solvent. An acrylic opaque resin (Super-Bond C $\&$ B, Sun Medical) was used as a bonding agent. A lightactivated microfilled indirect composite (New Metacolor Infis, Sun Medical Co., Ltd.) was employed as a veneering agent. A gold casting alloy (Casting Gold M. C. Type IV, GC Corp., Tokyo, Japan) was selected as the substrate material. Information about the materials is summarized in Table 1.

Ninety-six disk specimens $(10 \mathrm{~mm}$ in diameter by 2.5 $\mathrm{mm}$ in thickness) were cast from the gold alloy according to the manufacturer's specifications. All disks were ground with 600-grit silicon-carbide abrasive paper, followed by air-abrasion with 50-70 $\mu \mathrm{m}$ alumina (Hi-Aluminas, Shofu, Kyoto, Japan). The air-pressure of the air-borne particle abrader (Jet Blast 2, J. Morita, Osaka, Japan) was 0.45 MPa and the distance to the orifice from the metal surface was 5-10 mm. A piece of double-coated tape with a circular

Table 1 Materials assessed

\begin{tabular}{|c|c|c|c|}
\hline Material/System & Trade name & Lot No. & Component/Information \\
\hline \multicolumn{4}{|l|}{ Casting alloy } \\
\hline \multicolumn{2}{|c|}{ Casting Gold M. C. Type IV } & 1201 & $70 \% \mathrm{Au}, 16 \% \mathrm{Cu}, 8 \% \mathrm{Ag}, 3 \% \mathrm{Pd}, 2 \% \mathrm{Pt}, 1 \%$ Others \\
\hline \multicolumn{4}{|c|}{ Metal conditioner } \\
\hline \multicolumn{2}{|l|}{ Infis Opaque Primer } & 80601 & VTD, Acetone \\
\hline \multicolumn{4}{|l|}{ Bonding agent } \\
\hline \multirow[t]{3}{*}{ Super-Bond C \& B } & Opaque Ivory powder & VM1 & Poly(methyl methacrylate) (PMMA), Titanium dioxide \\
\hline & Monomer Liquid & EF1 & 4-META, Methyl methacrylate (MMA) \\
\hline & Initiator & VX13 & Tri-n-butylborane (TBB) \\
\hline \multicolumn{4}{|l|}{ Indirect composite } \\
\hline \multirow[t]{2}{*}{ New Metacolor Infis } & Opaque Base/Top A3-O & $80610 \mathrm{~F}, \mathrm{TV} 2$ & \\
\hline & Dentin A3-B & ES1 & UDMA, TEGDMA, Pre-polymerized composite filler \\
\hline
\end{tabular}

VTD, 6-(4-vinylbenzyl- $n$-propyl) amino-1,3,5-triazine-2,4-dithiol, -dithione tautomer; 4-META, 4-methacryloyloxyethyl trimellitate anhydride; UDMA, Dimethacryloyloxyethyl 2,2,4- (or 2,4,4-) trimethylhexamethylene diurethane; TEGDMA, Triethyleneglycol dimethacrylate. Threecomponent Super-Bond C\&B resin and two-component unfilled Super-Bond C\&B resin without the Opaque Ivory powder were assessed in the experiment. 
hole of $5 \mathrm{~mm}$ in diameter was positioned on each disk metal specimen to define the bond area.

The disks were divided into six groups, each of which consisted of 16 specimens. Group 1 was left unprimed and was used as a control. Group 2 was unprimed, but an unfilled resin bonding agent (Mixture of Super-Bond monomer liquid and the TBB initiator) was applied. Group 3 was unprimed, but the three-component Super-Bond C $\&$ B resin was applied by the brush-dip technique as the bonding agent. Group 4 was simply primed with the Infis Opaque Primer material, and bonding agent was not applied. Group 5 was primed with the Infis Opaque Primer conditioner, followed by application of the unfilled resin bonding agent (Super-Bond monomer and TBB initiator). Group 6 was primed with the Infis Opaque Primer material, followed by application of Super-Bond C\&B resin as the bonding agent.

A thin layer of an opaque resin (New Metacolor Infis Opaque Base) was placed on each of the 96 metal specimens and light-exposed for 30 seconds in an oven (Alpha Light II, J. Morita). Two layers of an additional opaque material (New Metacolor Infis Opaque Top A3O) were next applied on top of the primary opaque material, with each layer light-exposed for 90 seconds in the same oven. After light-exposure of the opaque resins, a brass ring (inner diameter: $6 \mathrm{~mm}$, length: $2 \mathrm{~mm}$, wall thickness: $1 \mathrm{~mm}$ ) was placed around the opaque resin. The ring was filled with the New Metacolor Infis composite material (dentin color, A3-B), and exposed in the Alpha Light II oven for 10 minutes. Sixteen specimens were prepared for each conditioning group.

One hour after preparation, the specimens were immersed in $37^{\circ} \mathrm{C}$ water for 24 hours, and this state was defined as thermocycle 0 . Half of the specimens (six sets of eight specimens) were tested for 24-hour shear bond strength at thermocycle 0 . The remaining six sets of eight specimens were subsequently placed in a thermocycling apparatus (Rika Kogyo, Tokyo, Japan) and cycled between $4^{\circ} \mathrm{C}$ and $60^{\circ} \mathrm{C}$ water with a $1-$ minute dwell time per bath for 20,000 cycles.

Each specimen was embedded in a steel mold and seated in an ISO 11405 shear testing jig. Shear bond strengths were then determined using a mechanical testing machine (10 kNG, Shimadzu, Kyoto, Japan) at a crosshead speed of $0.5 \mathrm{~mm} /$ minute. For each condition, the average bond strength and standard deviation (SD) of eight specimens were calculated. The shear testing results were analyzed by Mann-Whitney's U-test with the value of statistical significance set at 0.05 .

\section{Results}

Primary statistical analysis indicated that prethermocycling bond strengths for group $4\left(\mathrm{chi}^{2}=4.67, P\right.$ $\left.=0.03, \operatorname{chi}^{2}(0.95)=3.84\right)$ as well as post-thermocycling bond strengths for group $1\left(\mathrm{chi}^{2}=5.09, P=0.02, \mathrm{chi}^{2}(0.95)\right.$ $=3.84$ ) were not categorized as having a normal distribution. The results were therefore analyzed by Mann-Whitney's $U$-test with the value of statistical significance set at 0.05 .

Table 2 summarizes the bond strengths for each group both before and after thermocycling. The greatest bond strength (34.2 MPa) was obtained for group 6 before thermocycling. The lowest bond strength (2.5 MPa) was recorded for group 1 after thermocycling, in which neither the primer nor the bonding agent was used. Pre-

'Table 2 Shear testing and statistical analysis results for thermocycle 0 and 20,000 cycles

\begin{tabular}{|c|c|c|c|c|c|c|c|c|}
\hline \multirow{2}{*}{\multicolumn{2}{|c|}{$\begin{array}{l}\text { Primer } \\
\text { Group }\end{array}$}} & \multirow{2}{*}{$\begin{array}{c}\text { Bonding agent } \\
\text { Shear bond strength }(\mathrm{MPa})\end{array}$} & \multicolumn{2}{|c|}{ Thermocycle 0} & \multicolumn{2}{|c|}{20,000 cycles } & \multirow[t]{2}{*}{ Reduction } & \multirow[t]{2}{*}{ Tied $P$-value } \\
\hline & & & Mean & $\mathrm{SD}$ & Mean & $\mathrm{SD}$ & & \\
\hline 1 & None & None & 16.4 & 2.5 & 2.5 & 1.4 & $84.8 \%$ & 0.0008 \\
\hline 2 & None & Unfilled Super-Bond & 19.7 & 1.9 & 6.3 & 0.9 & $68.0 \%$ & 0.0008 \\
\hline 3 & None & Super-Bond C\&B & 33.7 & 3.3 & 14.3 & 3.5 & $57.6 \%$ & 0.0008 \\
\hline 4 & Infis Opaque Primer & None & 19.8 & 1.8 & 8.1 & 1.4 & $59.1 \%$ & 0.0008 \\
\hline 5 & Infis Opaque Primer & Unfilled Super-Bond & 24.0 & 2.1 & 13.9 & 1.2 & $42.1 \%$ & 0.0008 \\
\hline 6 & Infis Opaque Primer & Super-Bond C\&B & 34.2 & 2.5 & 23.4 & 1.2 & $31.6 \%$ & 0.0008 \\
\hline
\end{tabular}

SD: standard deviation

Tied $P$-value: difference between the thermocycle 0 and 20,000 cycles. 
thermocycling results demonstrate that the bond strengths for the unprimed groups was ranked as follows: group 3 , group 2, and group $1(P=0.0008$ and 0.0209$)$, whereas the strengths for the primed groups were ranked as follows: group 6, group 5, and group $4(P=0.0008$ and 0.0046$)$. Application of the bonding agent was effective regardless of the use of the primer. In addition, the effect of the threecomponent Super-Bond C \& B bonding agent was superior to that of the unfilled two-component Super-Bond material. Although the bond strength of the six groups was significantly reduced by the application of thermocycling (31.6-84.8\%, $P=0.0008$ ), the ranking before thermocycling remained unchanged even after the application of thermal stress $(P=0.0008)$.

Next, post-thermocycling bond strengths were compared for identical bonding agent conditions. The results revealed that the application of the Infis Opaque Primer material enhanced bond strength for all conditions (group $1<$ group $4, P=0.0008$; group $2<$ group $5, P=0.0008$; group $3<$ group $6, P=0.0011)$. The use of the thione primer was effective in all post-thermocycling conditions regardless of the application of the bonding agent.

\section{Discussion}

Type IV gold casting alloys are currently used for removable partial denture frameworks, including the external crowns of cone-telescope dentures. The external crowns are subjected to repeated loading during mastication, as well as the bending and tensile stresses associated with the insertion and/or removal of the denture frameworks. Probability of fracture or detachment of the veneering agent from the substructure is therefore much higher for cone-telescope crowns than for regular facing crowns. Since porcelain facing is generally contraindicated for veneering cone-telescope crowns, indirect composites are applied to facial and occlusal surfaces as tooth-colored materials. It is important for patients, dentists, and technicians that the framework and the veneering agent are strongly bonded with an adhesive system in order to minimize possibility of fracture of the tooth-colored materials.

The current study was designed to evaluate the effect of a metal conditioning agent and adhesive bonding agents on the bond durability between a gold casting alloy and a microfilled indirect composite. Comparison of the postthermocycling bond strengths between groups 1 and 4 revealed that the effect of the Infis Opaque Primer material alone was not particularly excellent, although bond strength was improved by priming. This is probably due to the fact that there is room for improvement with respect to the bond durability of the VTD monomer contained in the Infis
Opaque Primer or V-Primer material in application to the combination of gold alloys and indirect composites $(13,17)$. The use of a different thione compound may be beneficial for the improving bonding noble metal alloys $(8,12-14)$.

The current study used TBB-initiated adhesive resins for the improvement of bond strength. This choice was based on the development of a TBB-initiated opaque resin (1) and a report concerning the bonding of indirect composite to gold alloy using TBB opaque resin (24). Considering laboratory technical procedures, this study attempted to use a mixture of the TBB initiator and the 4META MMA solution as an unfilled resin bonding agent. This extra application of the Super-Bond resin without the powder component is derived from the expectation for the application of a very thin layer of bonding agent before application of the light-polymerized opaque resin. Comparison of the post-thermocycling bond strengths between groups 1 and 2, or between groups 4 and 5, demonstrated that the use of unfilled TBB bonding agent was substantially effective. The reduction in bond strength after thermocycling for group 5 was $42.1 \%$, despite the combined application of the primer and the bonding agent.

The authors therefore used the three-component SuperBond material as the bonding agent. As shown by the results for group 6, the combined use of the Infis Opaque Primer and the Super-Bond C \& B materials exhibited the greatest post-thermocycling bond strength and reduction in bond strength after thermocycling was $31.6 \%$. Although there is further possibility for improvement of the bond strength between the gold alloy and the indirect composite, application of the bonding system shown in group 6 is recommended as the best adhesive system within the limitations of the current study. Further investigation concerning the combination of priming agent and TBBinitiated bonding agent is necessary in order to improve bonding between gold alloys and indirect composites.

\section{Acknowledgments}

This work was supported in part by a Grant-in-Aid for Scientific Research (B(2) 16390566, 2004) from the Japan Society for the Promotion of Science and by a Nihon University Multidisciplinary Grant for 2004 (04-017).

\section{References}

1. Matsumura H, Nakabayashi N (1988) Adhesive 4META/MMA-TBB opaque resin with poly(methyl methacrylate)-coated titanium dioxide. J Dent Res 67, 29-32

2. Mori K, Nakamura Y (1983) Study on triazine thiols V. Polymerization of 6-(4-vinylbenzyl propyl)amino1,3,5-triazine-2,4-dithiol on copper plates and their 
corrosion resistance. J Polym Sci Polym Lett Ed 21, 889-895

3. Atsuta M, Matsumura H, Tanaka T (1992) Bonding fixed prosthodontic composite resin and precious metal alloys with the use of a vinyl-thiol primer and an adhesive opaque resin. J Prosthet Dent 67, 296300

4. Matsumura H, Leinfelder KF (1993) Effect of an adhesive primer on the integrity of occlusal veneermetal interface and wear of composite resin veneered restorations. J Prosthet Dent 70, 296-299

5. Tanaka T, Kamada K, Matsumura H, Atsuta M (1995) A comparison of water temperatures for thermocycling of metal-bonded resin specimens. J Prosthet Dent 74, 345-349

6. Watanabe I, Matsumura H, Atsuta M (1995) Effect of two metal primers on adhesive bonding with type IV gold alloys. J Prosthet Dent 73, 299-303

7. Matsumura H, Tanaka T, Atsuta M (1997) Bonding of silver-palladium-copper-gold alloy with thiol derivative primers and tri-n-butylborane initiated luting agents. J Oral Rehabil 24, 291-296

8. Matsumura H, Shimoe S, Nagano K, Atsuta M (1999) Effect of noble metal conditioners on bonding between prosthetic composite material and silverpalladium-copper-gold alloy. J Prosthet Dent 81, 710714

9. Matsumura H, Taira Y, Atsuta M (1999) Adhesive bonding of noble metal alloys with a triazine dithiol derivative primer and an adhesive resin. J Oral Rehabil 26, 877-882

10. Moulin P, Degrange M, Picard B (1999) Influence of surface treatment on adherence energy of alloys used in bonded prosthetics. J Oral Rehabil 26, 413421

11. Moulin P, Picard B, Degrange M (1999) Water resistance of resin-bonded joints with time related to alloy surface treatment. J Dent 27, 79-87

12. Matsumura H, Kamada K, Tanoue N, Atsuta M (2000) Effect of thione primers on bonding of noble metal alloys with an adhesive resin. J Dent 28, 287293

13. Matsumura H, Yanagida H, Tanoue N, Atsuta M, Shimoe S (2001) Shear bond strength of resin composite veneering material to gold alloy with varying metal surface preparations. J Prosthet Dent 86, 315-319
14. Matsumura H, Atsuta M, Tanoue N (2002) Evaluation of two thione primers and composite luting agents used for bonding a silver-palladiumcopper-gold alloy. J Oral Rehabil 29, 842-846

15. Kajihara H, Suzuki S, Kurashige H, Minesaki Y, Tanaka T (2003) Bonding abutments to cast metal post/cores: comparison of pre-treatment effects. J Oral Rehabil 30, 119-124

16. Watanabe I, Hotta M, Watanabe E, Atsuta M, Okabe $\mathrm{T}$ (2003) Shear bond strengths of laboratory-cured prosthetic composite to primed metal surfaces. Am J Dent 16, 401-403

17. Shimoe S, Tanoue N, Yanagida H, Atsuta M, Koizumi H, Matsumura H (2004) Comparative strength of metal-ceramic and metal-composite bonds after extended thermocycling. J Oral Rehabil 31, 689-694

18. Matsumura H, Atsuta M (1996) Repair of an eightunit fixed partial denture with a resin-bonded overcasting: a clinical report. J Prosthet Dent 75, 594596

19. Monya Y, Matsumura H, Atsuta M (1998) A twostage resin-bonded fixed partial denture seated in conjunction with postextraction healing of the alveolar socket: a clinical report. J Prosthet Dent 80, 4-8

20. Shimizu H, Takahashi Y (1999) Fixed splinting device to be used without removing adjacent existing cast restorations. J Prosthet Dent 82, 231-232

21. Shimizu H, Habu T, Yanagida H (2002) Stabilization by splinting of an endodontically treated premolar and a minimally reduced vital canine with a resinbonded cast retainer: a clinical report. Int Chin J Dent 2, 121-125

22. Hikage S, Hirose Y, Sawada N, Endo K, Ohno H (2003) Clinical longevity of resin-bonded bridges bonded using a vinyl-thiol primer. J Oral Rehabil 30, 1022-1029

23. Tanoue N, Yanagida H, Matsumura H (2004) Use of resin-bonded fixed partial dentures as permanent retainers: a clinical report. Int Chin J Dent 4, 40-43

24. Matsumura H, Kawahara M, Tanaka T, Atsuta M (1991) Surface preparations for metal frameworks of composite resin veneered prostheses made with an adhesive opaque resin. J Prosthet Dent 66, 1015 